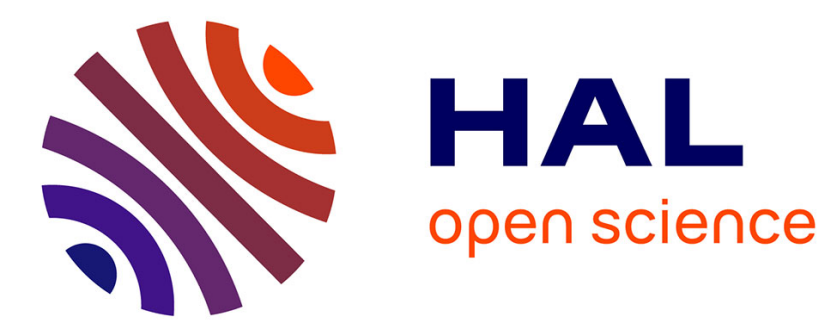

\title{
Broadband integrated polarization beam splitting based on anisotropic adiabatic transfer of light
}

\author{
Rim Alrifai, Virginie Coda, Andon A Rangelov, Germano Montemezzani
}

\section{To cite this version:}

Rim Alrifai, Virginie Coda, Andon A Rangelov, Germano Montemezzani. Broadband integrated polarization beam splitting based on anisotropic adiabatic transfer of light. Physical Review A, 2019, 100 (6), pp.063841. 10.1103/PhysRevA.100.063841 . hal-02425080

\section{HAL Id: hal-02425080 \\ https://hal.science/hal-02425080}

Submitted on 16 Apr 2020

HAL is a multi-disciplinary open access archive for the deposit and dissemination of scientific research documents, whether they are published or not. The documents may come from teaching and research institutions in France or abroad, or from public or private research centers.
L'archive ouverte pluridisciplinaire HAL, est destinée au dépôt et à la diffusion de documents scientifiques de niveau recherche, publiés ou non, émanant des établissements d'enseignement et de recherche français ou étrangers, des laboratoires publics ou privés. 


\title{
Broadband integrated polarization beam splitting based on anisotropic adiabatic transfer of light
}

\author{
Rim Alrifai, ${ }^{1,2, *}$ Virginie Coda $\odot,{ }^{1,2}$ Andon A. Rangelov, ${ }^{3}$ and Germano Montemezzani $\odot^{1,2}$ \\ ${ }^{1}$ LMOPS, Université de Lorraine, 2 Rue Édouard Belin, 57070 Metz, France \\ ${ }^{2}$ LMOPS, CentraleSupélec, 2 Rue Édouard Belin, 57070 Metz, France \\ ${ }^{3}$ Department of Physics, Sofia University, Boulevard James Bourchier 5, 1164 Sofia, Bulgaria
}

(Received 9 September 2019; published 23 December 2019)

\begin{abstract}
A concept for the realization of waveguide-based polarization beam splitters working over a wide spectral range is presented. The adiabatically evolving three-waveguide system is inspired by the quantum-mechanical stimulated Raman adiabatic passage process and involves waveguides with an anisotropic refractive index contrast. It is shown that an index contrast ratio of 5 is sufficient to obtain excellent efficiency and output polarization purity over a spectral range approximately equal to $350 \mathrm{~nm}$ centered at a wavelength of $1550 \mathrm{~nm}$. The center wavelength can be easily modified by tuning the parameters of the waveguides.
\end{abstract}

DOI: 10.1103/PhysRevA.100.063841

\section{INTRODUCTION}

Integrated polarization beam splitters (PBSs) play a significant role in many integrated photonic devices where the polarization state of light requires manipulation. Such components exploit birefringent waveguides to separate the input light into two orthogonal polarizations at the output (TE and TM). Demonstrations and proposals were based on directional couplers or $\mathrm{Y}$-junction structures with the inconvenience of a strong dependence on wavelengths [1-4], preventing them from being used, for instance, in wavelength multiplexing systems. Since then, considerable effort has been devoted to developing broadband PBSs, especially on the silicon-oninsulator platform exploiting the geometrical birefringence of waveguides realized by inducing a difference between the effective refractive index of the TE and TM modes [5]. This is mainly achieved due to high refractive index contrast and reduced dimensions. Various structures have been reported, e.g., highly birefringent bent couplers [6,7], asymmetric phase-controlled directional couplers [8,9], or multimode interference couplers sometimes combined with subwavelength gratings [10], photonic crystals [11], or metamaterials $[12,13]$ to extend the wavelength bandwidth. These devices can reduce the device footprint, generally in exchange for tight fabrication tolerances along with precise and complex tuning of the waveguide structures.

Here we focus on relatively simple and extremely broadband PBSs using adiabatic directional coupler structures which use quantum-mechanical analogies [14]. A very popular and useful adiabatic phenomenon in quantum physics is the stimulated Raman adiabatic passage (STIRAP) process that applies in its original form to the transfer of population within a three-state discrete quantum system [15]. It allows the robust and highly efficient population transfer from the initial to the final state without populating the intermediate state. A STIRAP-like transfer in optics consists in a system

\footnotetext{
*rim.alrifai@univ-lorraine.fr
}

formed by an odd number of waveguides to transfer light from the first waveguide to the last one [16,17]. Such an approach is intrinsically robust with respect to the system parameters. Specifically, it permits broadband operation. Such analogies have been successfully used to experimentally demonstrate broadband light transfer [18-21].

Combining the above STIRAP-like light transfer with waveguides showing a different propagation constant for the $\mathrm{TE}$ and TM polarizations can be exploited advantageously to realize an integrated PBS [22,23]. In the present work we propose an alternative and simple method, where no precise adjustment of the transverse shapes and dimensions of the waveguides is required to induced the difference in the TE and $\mathrm{TM}$ effective refractive indices. The idea is to exploit directly the anisotropy of material. Few attempts have exploited material anisotropy to realize integrated PBSs, and the systems often require the integration of different materials in distinct parts or sections of the coupler $[2,23,24]$, which increases the difficulty of the technological process. Here we demonstrate that a three-waveguide system can be built with the same anisotropic refractive index contrast for all waveguides.

We use systems composed of three waveguides whose mutual coupling depends on the guided light polarization (TE or TM) and varies adiabatically along the propagation direction $z$ due to a variation of the distance between the individual waveguides (see Fig. 1). The two outer waveguides are used as output ports and the central one is an auxiliary waveguide. Section II elucidates the theoretical background for the wave propagation in the STIRAP-like anisotropic waveguide system. Section III gives several examples of the expected performance of such devices as calculated using the coupled-mode theory (CMT) [25] and confirmed by a beam propagation method (BPM) [26]. The examples given in Sec. III are for a target central wavelength of $1550 \mathrm{~nm}$ and for the case where the index contrast associated with the TM wave is larger that the one for the TE wave. However, other target wavelengths or the reverse index contrast can be addressed similarly. Section IV discusses the results and the possible implementation methods. Section V summarizes. 


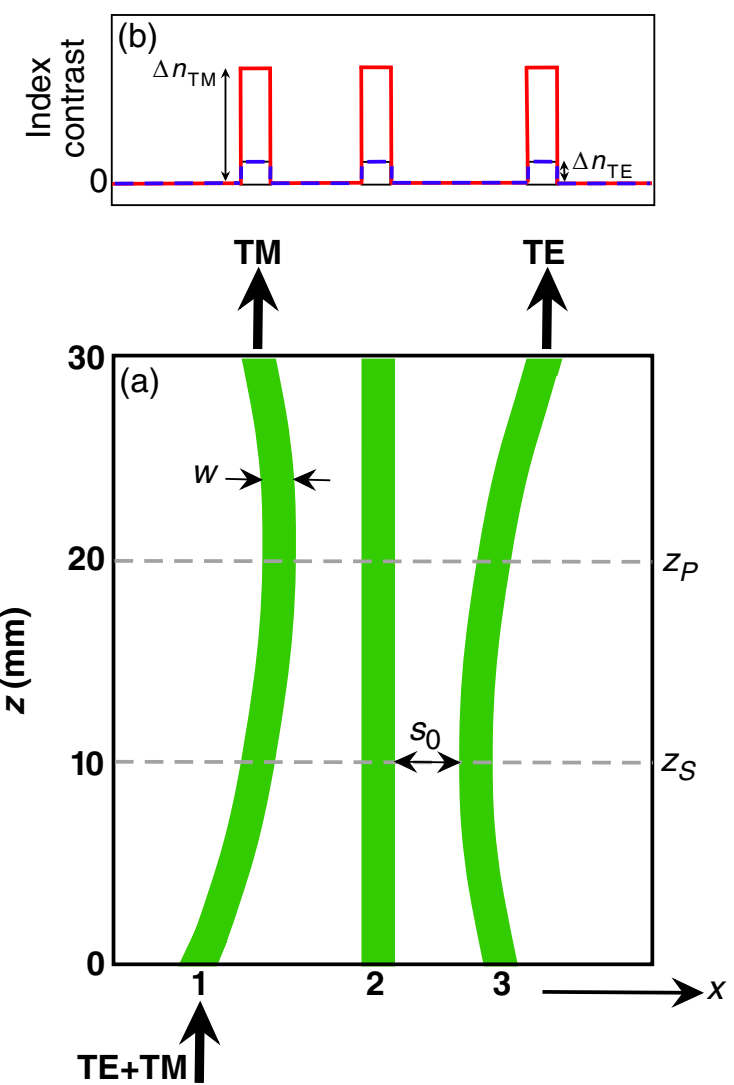

FIG. 1. (a) Waveguide structure for the broadband splitting of the TE and TM eigenpolarizations into two different output waveguides. The identical but differently shaped waveguides have a constant core width $w$ and a separation $s$ that depends on the propagation coordinate $z$. Here $z_{P}\left(z_{S}\right)$ is the position of smallest separation $s_{0}$ between the first (last) two waveguides. (b) Anisotropic refractive index contrast $\Delta n$ for the three step-index waveguides. Here the index contrast is supposed to be larger for the TM wave than for the TE wave.

\section{THEORETICAL BACKGROUND}

We consider a system of three waveguides arranged in the way shown in Fig. 1(a) and able to support both the fundamental TE and the TM modes. For the sake of simplicity, in the calculation presented in Sec. III the waveguides will be considered to be slab waveguides with a step-index contrast [see Fig. 1(b)], the step height being larger for the TM than for the TE polarization of the guided wave. However, the expressions given below hold generally independently of the form of the waveguides. In the framework of the CMT [25], the propagation of the electric-field amplitudes $a_{1}(z), a_{2}(z)$, and $a_{3}(z)$ of the waves traveling in the waveguide array is described by a system of three coupled differential equations written in matrix form as

$$
i \frac{d}{d z}\left[\begin{array}{l}
a_{1} \\
a_{2} \\
a_{3}
\end{array}\right]=\left[\begin{array}{ccc}
0 & C_{P}(z) & 0 \\
C_{P}(z) & 0 & C_{S}(z) \\
0 & C_{S}(z) & 0
\end{array}\right]\left[\begin{array}{l}
a_{1} \\
a_{2} \\
a_{3}
\end{array}\right],
$$

where $C_{P}(z) \equiv \sqrt{C_{12} C_{21}}$ and $C_{S}(z) \equiv \sqrt{C_{23} C_{32}}$ are the effective coupling coefficients between waveguides 1 and 2 and waveguides 2 and 3 , respectively. Here $C_{i j}$ is the coupling constant of waveguide $i$ to waveguide $j$ and in our case $C_{i j}=C_{j i}$ because the waveguides are supposed to be identical (the same form and same refractive index contrast for a given polarization). The subscripts $P$ and $S$ mean pump and Stokes, in analogy with the pump and Stokes pulses used in the conventional quantum STIRAP process [15]. This analogy stems from the fact that Eq. (1) is fully equivalent to the Schrödinger equation that describes the state population dynamics for a three-level quantum system coupled by two resonant pulses $[14,15,17]$.

The above system of equations (1) is in the so-called diabatic basis where the basis vectors $\mathbf{A}_{1}=(1,0,0), \mathbf{A}_{2}=$ $(0,1,0)$, and $\mathbf{A}_{3}=(0,0,1)$ (associated with the three waveguides) are fixed throughout the length of the device. However, since the Hamiltonian-like matrix in (1) evolves along the coordinate $z$, it is possible to transform it in a new basis (the adiabatic basis) where the basis vectors correspond to its local eigenvectors [27]. One of these eigenstates is the adiabatic transfer state or dark state that involves only the diabatic states $\mathbf{A}_{1}$ and $\mathbf{A}_{3}$,

$$
\mathbf{B}_{0}(z)=\mathbf{A}_{1} \cos \vartheta(z)-\mathbf{A}_{3} \sin \vartheta(z),
$$

with $\tan \vartheta(z) \equiv C_{P}(z) / C_{S}(z)$. For a STIRAP-like robust light transfer from waveguide 1 to waveguide 3 the system will remain always in this spatially evolving state in such a way that $\mathbf{B}_{0}(z=0)=\mathbf{A}_{1}$ and $\mathbf{B}_{0}(z=L)=-\mathbf{A}_{3}$, where $L$ is the length of the device. This means $\vartheta(0) \approx 0$ and $\vartheta(L) \approx \pi / 2$. It is easy to see that this requires a counterintuitive sequence of coupling constants $C_{P}$ and $C_{S}$. In practice, this means that $C_{S}$ is initially larger than $C_{P}$ and that the point $z_{S}$ at which the waveguides 2 and 3 are the closest (the point with the largest Stokes coupling constant $C_{S \text {, max }}$ ) is closer to the input than the corresponding point $z_{P}$ for the pump coupling constant. By a proper choice of the shape of the waveguides it can be assumed that the coupling coefficients have a Gaussian evolution along the propagation coordinate $z$,

$$
C_{P, S}(z)=C_{0} \exp \left[-\left(\frac{z-z_{P, S}}{\Delta z}\right)^{2}\right],
$$

where $\Delta z$ is the distance from $z_{P, S}$ at which the coupling constants fall to a value of $C_{0} / e$, with $C_{0}=C_{S \text {, max }}=C_{P, \max }$ the maximum value. In the framework of the coupled-mode theory, the coupling constant for identical slab-type waveguides of width $w$ and separation distance $s$ (center-to-center distance $w+s$ ) can be expressed as a function of the waveguide and mode parameters as [28]

$$
C=\frac{2 h^{2} \gamma \exp (-\gamma s)}{\beta(w+2 / \gamma)\left(h^{2}+\gamma^{2}\right)},
$$

where $\beta$ and $h$ are the longitudinal and transverse mode propagation constants, respectively, and $\gamma$ is the evanescent wave extinction coefficient. Note that for a given waveguide geometry the three above parameters depend on light wavelength. The dominant term in the above equation is the exponential term in the numerator, which leads to a strong decrease of $C$ upon increase of the separation distance $s$. This allows us to adjust the $z$ dependence of $C_{P}$ and $C_{S}$ in Eq. (3) by adjusting the waveguides shape, which is the function $s(z)$ for each pair of waveguides. 
It is worth noting that Eq. (4) is derived analytically by integrating the overlap-type integral issued from the perturbational approach, which is at the base of the coupled-mode theory for the case of a TE mode [28]. However, within the range of parameters used for our study, the numerical integration of the corresponding overlap integral for TM modes delivers values consistent with Eq. (4). This shows that in our considered regime this expression can be applied to TM modes as well. As discussed below, in our case the wanted for strong difference in the coupling constants for the two orthogonal polarization arises fully from a different waveguide index contrast $\Delta n$. Indeed, since the quantities $\beta, h$, and $\gamma$ depend on $\Delta n$, in the case where the latter is anisotropic, Eq. (1) splits into two equations because the two eigenmode polarizations can be associated with very different values of $C_{P}$ and $C_{S}$, for instance, $C_{P, \mathrm{TE}} \gg C_{P, \mathrm{TM}}$ and $C_{S, \mathrm{TE}} \gg C_{S, \mathrm{TM}}$. Obviously $\beta$, $h$, and $\gamma$ differ slightly between the TE and TM waves even in the case of a common (isotropic) refractive index contrast. However, in this case the effect on the coupling constants and on the output intensity distribution for a waveguide system such as the one of Fig. 1(b) is only marginal.

In order to separate the polarizations into two different output ports (waveguides 1 and 3 ) one should therefore ensure that for one polarization (in our examples TE) one obtains a STIRAP-like adiabatic light transfer from the input waveguide [waveguide 1 (WG1)] to waveguide 3 (WG3), while for the other polarization this does not occur and the TM light exits WG1. The light transfer is efficient if the above dark state $\mathbf{B}_{0}$ does not couple to the two other adiabatic states. This is satisfied if the coupling outer-diagonal terms in the adiabatic version of the matrix in Eq. (1) are sufficiently small. It can be shown that this is fulfilled if the system satisfies the adiabatic condition that states that the quantity $\left(C_{P, \max }^{2}+C_{S, \max }^{2}\right)^{1 / 2}$ should greatly exceed the reciprocal of the overlap distance between the functions $C_{P}(z)$ and $C_{S}(z)$ [see Ref. [27] and Eq. (8) in Ref. [20]]. Therefore, since in our case we have $C_{S, \max }=C_{P, \max }=C_{0}$, the nature of light transfer is controlled essentially by the value of $C_{0}$, which, for a given waveguide geometry, depends on the refractive index contrast $\Delta n$ and on the wavelength $\lambda$.

The procedure for the simulations of the broadband beam splitting behavior involves several steps. After setting the length of the structure to $30 \mathrm{~mm}$, for every choice of the parameters of the waveguides (core width $w$ and the minimum separation $s_{0}$ ), the shape of the two outer waveguides is adjusted in such a way to get an as good as possible adiabatic light transfer while fulfilling the function (3) for a particular value of $\Delta n_{\mathrm{TE}}$ and wavelength $\lambda$ (generally $\lambda=1550 \mathrm{~nm}$ ). At this point the waveguide shape is no longer modified and the functions $C_{P}(z)$ and $C_{S}(z)$ are calculated for both the TE and the TM waves for various values of $\lambda$ and of the refractive index contrast $\Delta n$. These functions are then used to integrate the mode amplitude spatial evolution [Eq. (1)] and to obtain the output intensities in the three waveguides $\left(I_{i} \propto\left|a_{i}\right|^{2}\right)$ for both the TE and TM waves under the initial condition that both polarizations are injected into WG1. The results obtained by this method based on coupled-mode theory are compared with numerical simulations by the BPM in waveguides having the same shape and the same index contrast. The results are given in the next section.
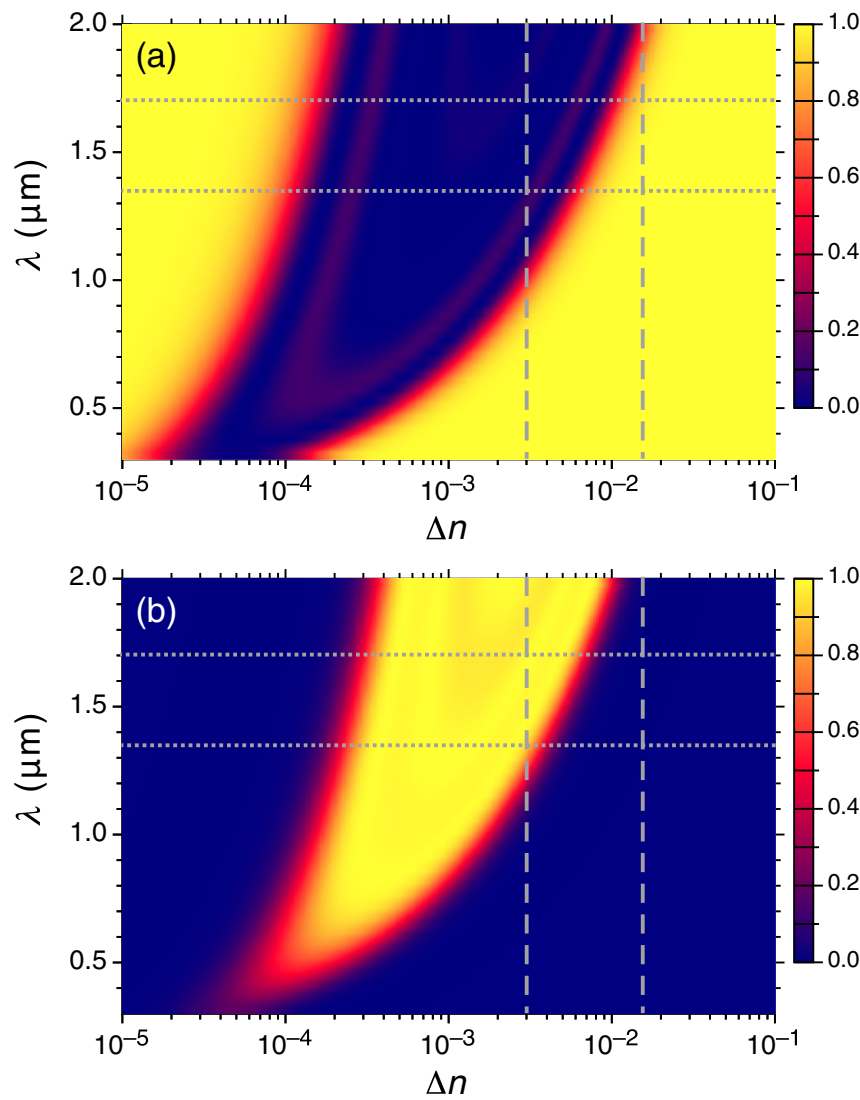

FIG. 2. (a) Color plot of the output intensity $I_{1}$ in WG1 as a function of refractive index contrast $\Delta n$ and wavelength $\lambda$. (b) Corresponding output intensity $I_{3}$ in WG3. Light is input into WG1. The waveguide parameters $w, s_{0}$, and $L$ are given in the text. The diagrams hold for both TE and TM polarization. The simulations are done by the coupled-mode theory [integration of Eq. (1)] as described in the text.

\section{BROADBAND POLARIZATION BEAM SPLITTING}

It is useful to first orient the search for appropriate parameters with the help of diagrams such as those in Fig. 2 obtained by the procedure described above. Here the output intensities in WG1 [Fig. 2(a)] and WG3 [Fig. 2(b)] are represented as color plots as a function of $\Delta n$ and $\lambda$ for the case where a TE wave is injected in WG1. Note that, due to the small index contrast and the considered dimensions, the corresponding plots for a TM input wave are essentially indistinguishable from these. In Fig. 2 we have chosen a total structure length $L$ of $30 \mathrm{~mm}$, a core width $w=5 \mu \mathrm{m}$, and a minimum separation distance $s_{0}=7 \mu \mathrm{m}$. Note that varying these parameters permits us to tune the operation conditions of the component and its spectral characteristics. For instance, increasing the value of $w$ shifts the blue region in Fig. 2(a) and the yellow region in Fig. 2(b) towards the left because, due to the better mode confinement, a strong enough coupling coefficient would be found for smaller values of $\Delta n$. Similarly, a larger minimum separation would have the same effect.

The landscape of Fig. 2 permits an easy orientation of the values of the refractive index contrasts required for a good separation of the orthogonal polarizations. Let us look 
for a pair of index contrasts ( $\Delta n_{\mathrm{TE}}$ and $\Delta n_{\mathrm{TM}}$ ) leading to very different behavior at the output in the spectral range of interest, which is in our example the band between the two horizontal dotted lines around $1.5-\mu \mathrm{m}$ wavelength. For the smallest $\Delta n$, in our case associated with $\Delta n_{\mathrm{TE}}$, the light should pass from WG1 to WG3, meaning that we should be in the blue region in Fig. 2(a) and in the yellow region in Fig. 2(b). In the spectral range of interest, this is well satisfied for a $\Delta n_{\mathrm{TE}}=0.003$ corresponding to the left vertical dashed line. For the other polarization, associated with a larger index contrast, we require that the light stays in WG1 and should therefore be in the yellow region of Fig. 2(a) and blue region of Fig. 2(b). This is the case for an index contrast $\Delta n_{\mathrm{TM}}=$ 0.015 , five times larger than $\Delta n_{\mathrm{TE}}$ and corresponding to the right dashed vertical line. Note that for this value of $\Delta n_{\mathrm{TM}}$, for wavelengths exceeding $1.7 \mu \mathrm{m}$, even though no light reaches WG3, part of the light leaves WG1 and couples to WG2 (orange region). Note also that the same reasoning as above can be made for other spectral ranges. For instance, for the spectral range around $1.0 \mu \mathrm{m}$ and the same parameters $w, s_{0}$, and $L$ as above, we would need $\Delta n_{\mathrm{TE}} \approx 0.001$ and a roughly five times larger $\Delta n_{\mathrm{TM}}$. We have chosen not to exploit the frontier on the left part of the diagrams in Fig. 2, which corresponds to small $\Delta n$. One can notice that in these regions, the frontier between the yellow and the blue regions for $I_{1}$ and $I_{3}$ has a much bigger $\Delta n$ shift than the right frontier. This is due to the weaker horizontal gradient in the low $\Delta n$ region, which would imply the necessity of a much stronger refractive index contrast anisotropy than for the examples given below. Moreover, these small $\Delta n$ correspond to poor mode confinements, for which the CMT approximation is not fully valid anymore.

Based on the above discussion, we select a value of $\Delta n_{\mathrm{TE}}=0.003$ and concentrate first on the intensity evolution in the three waveguides for the central wavelength of $1550 \mathrm{~nm}$. This is shown in Fig. 3, which depicts the wave propagation in the three-waveguide structure for different values of the index contrast. Figures 3(a) and 3(d) are for the TE wave and the above index contrast. Clearly, in this case the wave is coupled during the propagation to the other waveguides and exits from WG3 with only very little transient light in WG2. This beam evolution is characteristic of an adiabatic and thus robust transfer and close to the one typical for a STIRAP-like light transfer. Figures 3(b) and 3(e) are for the TM wave and a five times larger index contrast $\Delta n_{\mathrm{TM}}=0.015$. In this case the coupling is inhibited and the light remains in the first waveguide all along with only very little light coupling to WG2. Finally, the confinement of the TM wave in WG1 is even more effective in the case where the index contrast ratio $\Delta n_{\mathrm{TM}} / \Delta n_{\mathrm{TE}}$ is increased further to roughly 7.5 as in Figs. 3(c) and 3(f). Figure 3 confirms that for $\lambda=1550 \mathrm{~nm}$ the wished for separation of polarizations is well achieved for an index contrast ratio $\Delta n_{\mathrm{TM}} / \Delta n_{\mathrm{TE}}=5$, which is consistent also with the BPM simulations.

Figure 2 also clearly shows that the beam splitting behavior seen in Fig. 3 is expected to fail at wavelengths shorter than $1 \mu \mathrm{m}$ if the same waveguide parameters are used. Before going on to discuss the spectral dependence in more detail, it is useful to define some quality parameters for the beam splitters. The first one $\xi_{1}$ is the purity of the TM wave
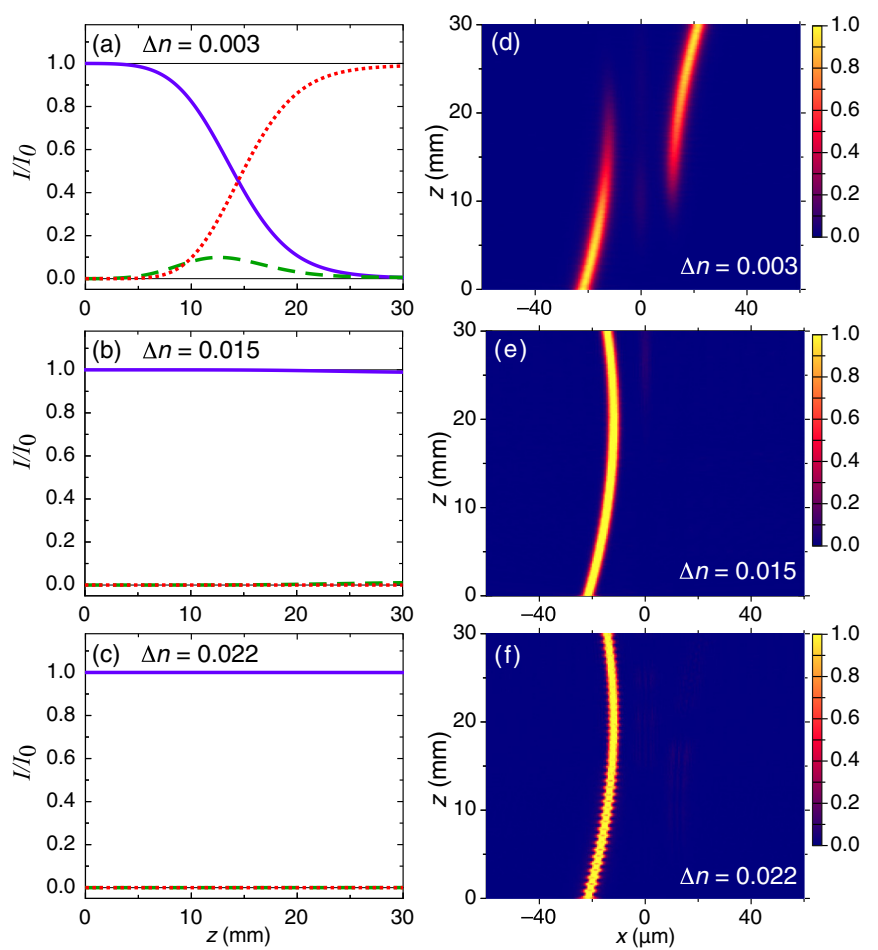

FIG. 3. Spatial evolution of the intensity in the three waveguides for $\lambda=1550 \mathrm{~nm}$ and the same waveguide parameters $w$ and $s_{0}$ used for Fig. 2, calculated using (a)-(c) the CMT (1) and (d)-(f) the BPM for (a) and (d) $\Delta n=0.003$ associated with the TE wave exiting WG3, (b) and (e) $\Delta n=0.015$ associated with the TM wave, and (c) and (f) $\Delta n=0.022$ associated with the TM wave. In (a)(c) the blue solid curve gives the intensity $I_{1}(z)$ in WG1, while the green dashed curve and the red dotted curve give $I_{2}(z)$ and $I_{3}(z)$, respectively.

remaining in WG1, which is the ratio of the intensity of the TM wave and the total intensity (TM plus TE) in this same waveguide,

$$
\xi_{1}=\frac{I_{1, \mathrm{TM}}}{I_{1, \mathrm{TM}}+I_{1, \mathrm{TE}}} .
$$

The second one is the corresponding purity $\xi_{3}$ of the TE wave in WG3

$$
\xi_{3}=\frac{I_{3, \mathrm{TE}}}{I_{3, \mathrm{TM}}+I_{3, \mathrm{TE}}},
$$

while the last one is the efficiency of the system defined as

$$
\eta=\sqrt{I_{1, \mathrm{TM}} I_{3, \mathrm{TE}}} .
$$

All intensities in Eqs. (5)-(7) are taken at the output of the system $(z=L)$ and a unity input intensity is assumed for Eq. (7).

The three above quality factors are plotted in Fig. 4 as a function of the refraction index contrast ratio for three wavelengths, 1400, 1550, and $1700 \mathrm{~nm}$. Note that here the refractive index contrast of the TE wave (and therefore its coupling behavior) is kept constant and only $\Delta n_{\mathrm{TM}}$ is changed. It can be seen that already for a ratio $\Delta n_{\mathrm{TM}} / \Delta n_{\mathrm{TE}}=3$ the two polarizations are well separated $\left(\xi_{1} \approx 1\right.$ and $\left.\xi_{3} \approx 1\right)$ for $\lambda=1550 \mathrm{~nm}$, while the system is not yet efficient enough 

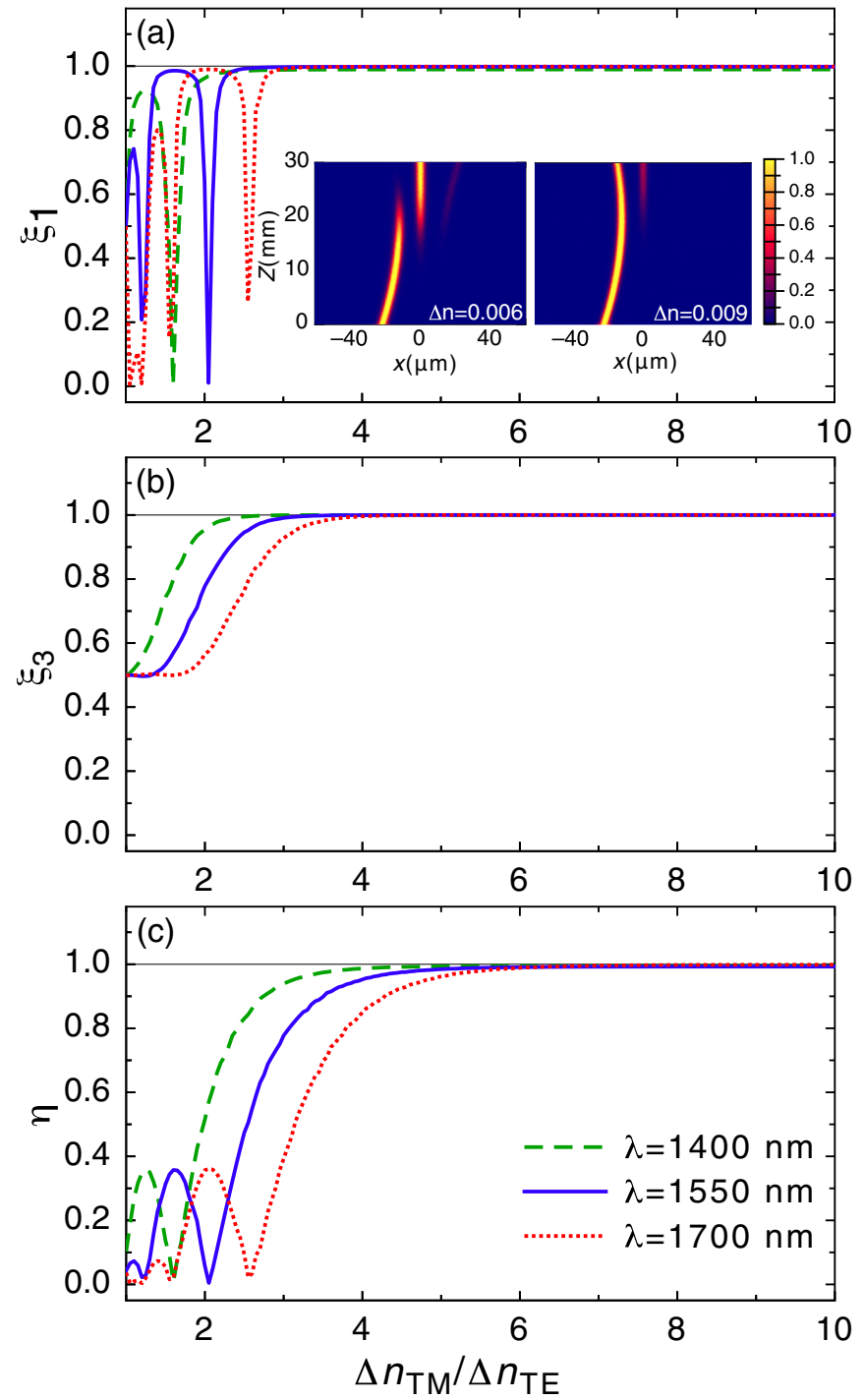

FIG. 4. (a) Purity $\xi_{1}$ of the TM polarized light at the output of WG1 and (b) corresponding purity $\xi_{3}$ of the TE wave at the output of WG3. (c) Efficiency $\eta$ of the polarizing beam splitter. All quantities are plotted as a function of the refractive index contrast ratio $\Delta n_{\mathrm{TM}} / \Delta n_{\mathrm{TE}}$ for wavelengths of $1400 \mathrm{~nm}$ (green dashed line), $1550 \mathrm{~nm}$ (blue solid line), and $1700 \mathrm{~nm}$ (red dotted line); $\Delta n_{\mathrm{TE}}=$ 0.003 is fixed. The insets in (a) show the BPM propagation for $\lambda=1.55 \mu \mathrm{m}$ for $\Delta n_{\mathrm{TM}}=0.006$ and 0.009 .

because a small part of the TM polarized light is transferred to WG2. This is shown in the right-hand inset in Fig. 4(a), giving the BPM results for $\Delta n=0.009$. This effect is even stronger for $\Delta n_{\mathrm{TM}} / \Delta n_{\mathrm{TE}}=2$, corresponding to the left inset in Fig. 4(a). In this case, most of the TM wave exits WG2 and the remaining light in WG1 is even weaker than the spurious TE light remaining in this waveguide [see Fig. 3(a)] so, the purity $\xi_{1}$ gets near a minimum. In general, the oscillations seen in the quantities $\xi_{1}$ and $\eta$ for $\Delta n_{\mathrm{TM}} / \Delta n_{\mathrm{TE}}<3$ are due to the fact that the refractive index contrast of the TM wave is not large enough to lead to a full confinement of the TM wave in WG1, but it is not small enough to lead to an adiabatic light passage of this wave to WG3. For $\Delta n_{\mathrm{TM}} / \Delta n_{\mathrm{TE}}>3$ all the curves in Fig. 4 become more gentle. Again, it can be
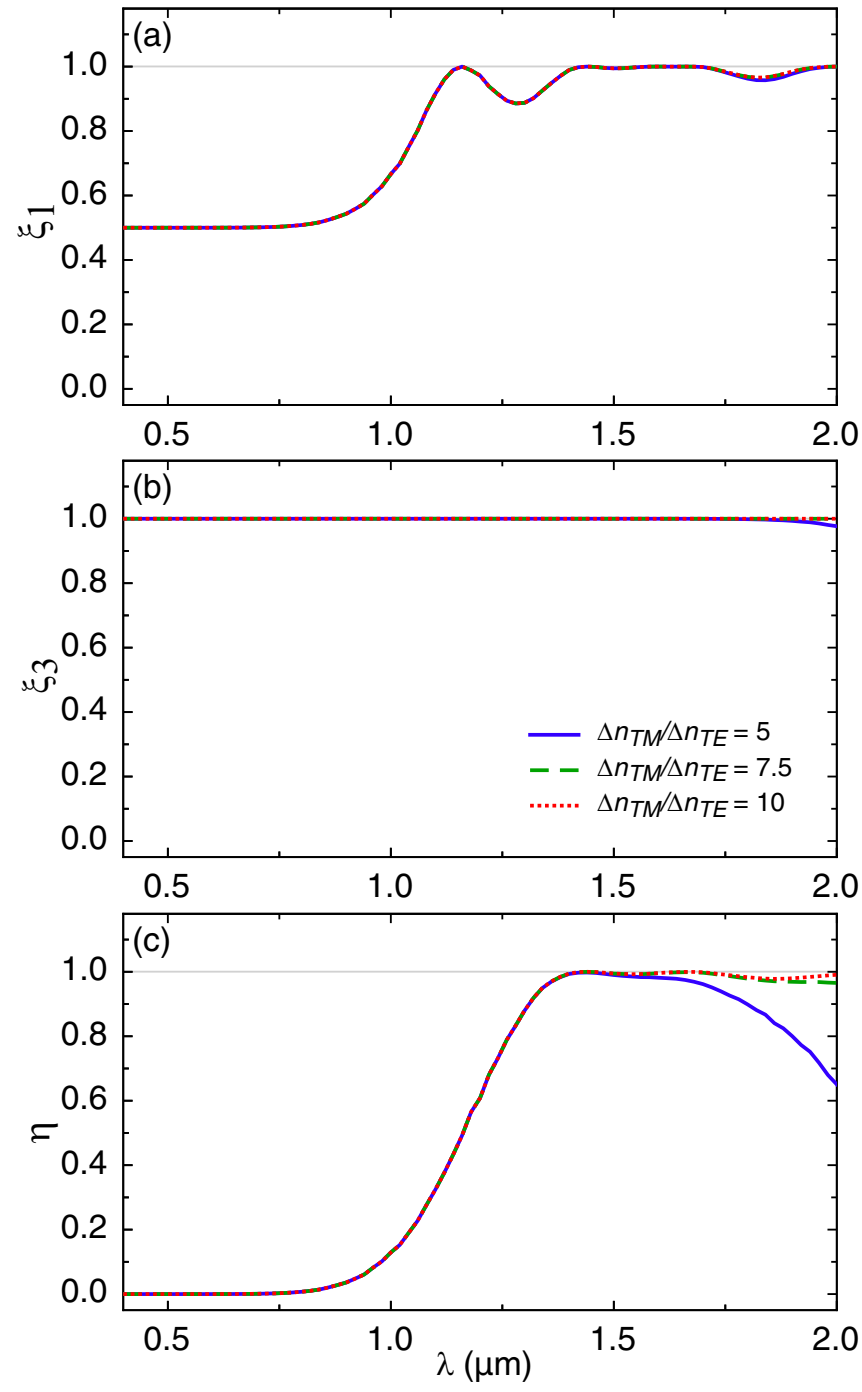

FIG. 5. (a) Purity $\xi_{1}$ of the TM polarized light at the output of WG1 and (b) corresponding purity $\xi_{3}$ of the TE wave at the output of WG3. (c) Efficiency $\eta$ of the polarizing beam splitter. All quantities are plotted as a function of the wavelength $\lambda$ for $\Delta n_{\mathrm{TM}} / \Delta n_{\mathrm{TE}}=5$ (blue solid line), 7.5 (green dashed line), and 10 (red dotted line); $\Delta n_{\mathrm{TE}}=0.003$ is fixed.

concluded that a ratio $\Delta n_{\mathrm{TM}} / \Delta n_{\mathrm{TE}}=5$ gives a good polarizing beam splitter for $\lambda=1550 \mathrm{~nm}$ for which all quality factors are near unity. The most critical quantity is the efficiency $\eta$. It can be seen in Fig. 4(c) that shorter wavelengths can afford a smaller refractive index contrast ratio than longer ones to get a good efficiency.

Next the quality factors $\xi_{1}, \xi_{3}$, and $\eta$ are represented in Fig. 5 as a function of wavelength for three index contrast ratios. Figure 5(b) shows that the purity $\xi_{3}$ of the TE wave is always very good and Fig. 5(a) shows that the purity of the TM wave has the same spectral dependence regardless of the value of $\Delta n_{\mathrm{TM}} / \Delta n_{\mathrm{TE}}$. This is due to the fact that, even though the TM light is always well confined in WG1, the value of $\xi_{1}$ is mainly governed by the part of the TE wave remaining in WG1. For instance, for $\lambda<0.8 \mu \mathrm{m}$ and $\Delta n_{\mathrm{TE}}=0.003$ most of the TE wave stays in WG1 (no adiabatic light passage occurs) and the purity $\xi_{1}$ falls to about $1 / 2$. This also explains 
the efficiency drop on the short-wavelength side in Fig. 5(c) because, due to the weaker evanescent coupling, the TE wave does not make the adiabatic passage any longer and eventually does not reach WG3 with a sufficient intensity. On the other hand, on the long-wavelength side, there is a slight decrease in $\eta$ due to the fact that the TM wave gets poorer confinement in WG1. The efficiency bandwidth on the long-wavelength side can be easily increased by increasing the index contrast ratio, as shown in Fig. 5(c).

\section{DISCUSSION}

The example given above shows that a system composed of three waveguides with anisotropic refractive index contrast with a ratio of about 5 between the TM and TE polarization is able to give a very broadband integrated polarizing beam splitter with nearly unit purity and efficiency over a spectral range of approximately $350 \mathrm{~nm}$ around the 1550-nm telecommunication wavelength. Larger index contrast ratios lead to a further increase of this bandwidth. These results can be easily transposed to other spectral ranges by simply modifying the waveguide parameters (width, minimum separation, and refractive index contrast of the less confined wave), as discussed in connection with Fig. 2.

Waveguides with an anisotropic refractive index contrast may be produced using several methods and a few examples where the required ratio of the index contrast will be achievable are given below. In principle, one might aim at different combinations, a waveguide core with an anisotropic refractive index combined with an isotropic cladding, an isotropic core and anisotropic cladding, or the case where both core and cladding possess an anisotropic refractive index with a different degree of anisotropy.

As an example, we may consider $\mathrm{LiNbO}_{3}$, which is one of the most used materials in integrated optics $[29,30]$ and is a uniaxial anisotropic crystal. In this material, the most common techniques for realizing optical waveguides are $\mathrm{Ti}$ ion in-diffusion [31] and proton exchange [32]. While Ti in-diffusion leads to a (slightly anisotropic) increase of both the extraordinary $\left(n_{e}\right)$ and the ordinary $\left(n_{o}\right)$ refractive indices, proton exchange leads to a stronger increase of $n_{e}$ and to a decrease of $n_{o}$. A combination of the two techniques will therefore permit one to adjust the ratio of the index contrast potentially to any given value. Such a combination of $\mathrm{Ti}$ diffusion and proton exchange has already been successfully tested in the past for other purposes [33-37].

Another option is to use waveguides made of polymers [38]. In this case polymers possessing a strong alignment birefringence, such as polyimides [39] or other strongly anisotropic compounds [40], or possessing a stress birefringence [41] or a photoinduced birefringence [42] are of particular interest. Suppose that a birefringent polymer with $n_{e}>n_{o}$ is used as the waveguide core and is surrounded by a cladding polymer with an isotropic refractive index $n$ only slightly smaller than $n_{o}$; then, in principle, extremely large ratios of the index contrasts for the TM and TE waves can be achieved.

Yet another possibility would be to take advantage of photoinduced waveguides in materials exhibiting the photorefractive effect [43-45] and allowing a postfixing process $[46,47]$. In this case, the primary effect is the creation of a local space-charge electric field; therefore, the anisotropy of the refractive index contrast is directly proportional to the ratio of the relevant electro-optic coefficients that translate the internal electric field into a change of refractive index by the Pockels effect. While in $\mathrm{LiNbO}_{3}$ the relevant ratio of the most important diagonal unclamped coefficients $r_{333}^{T} / r_{113}^{T}$ is only about 3 [48], other materials exhibit larger ratios, for instance, $r_{333}^{T} / r_{223}^{T} \approx 10$ in $\mathrm{KNbO}_{3}$ [49] and $r_{333}^{T} / r_{113}^{T} \approx 13$ in $\mathrm{BaTiO}_{3}$ [50].

While all the above proposals involve waveguide cores with an anisotropic refractive index, there is also the final possibility to use an isotropic core, such as a glass ridge or rib waveguide, covered by an anisotropic material acting as cladding. For instance, the latter may be again formed by a birefringent polymer with its largest refractive index only slightly lower than the one of the core material and allowing so potentially very large index contrast ratios which are only limited by the choice of the materials. In the framework of liquid crystal photonics [51], another quite appealing possibility in this context would be to use weakly birefringent liquid crystals as the cladding material. This would open the possibility for an electrical or thermo-optical control and switching of the optical properties of the cladding and of the beam splitting behavior. Model calculations for the anisotropic waveguide modes in such a configuration were performed by Beeckman et al. [52] and the tuning capability of such an approach in the case or ring resonators on a silicon-on-insulator platform was already demonstrated [53].

\section{CONCLUSION}

We have proposed an simple concept to realize broadband integrated polarization beam splitters based on a adiabatically evolving waveguide structure inspired by the quantum STIRAP process. The system is characterized by an anisotropic refractive index contrast of the three involved waveguides. Several approaches and materials may be selected to realize such optically anisotropic waveguides.

\section{ACKNOWLEDGMENT}

This work was partially supported by the Bulgarian Science Fund Grant No. DN 18/14.
[1] O. Mikami, Appl. Phys. Lett. 36, 491 (1980).

[2] N. Goto and G. L. Yip, J. Lightw. Technol. 7, 1567 (1989).

[3] I. Kiyat, A. Aydinli, and N. Dagli, IEEE Photon. Technol. Lett. 17, 100 (2005).
[4] S. Rojas-Rojas, L. Morales-Inostroza, U. Naether, G. B. Xavier, S. Nolte, A. Szameit, R. A. Vicencio, G. Lima, and A. Delgado, Phys. Rev. A 90, 063823 (2014).

[5] D. Dai, J. Bauters, and J. E. Bowers, Light Sci. Appl. 1, e1 (2012). 
[6] H. Fukuda, K. Yamada, T. Tsuchizawa, T. Watanabe, H. Shinojima, and S.-i. Itabashi, Opt. Express 16, 4872 (2008).

[7] H. Wu, Y. Tan, and D. Dai, Opt. Express 25, 6069 (2017).

[8] Z. Lu, Y. Wang, F. Zhang, N. A. F. Jaeger, and L. Chrostowski, Opt. Express 23, 29413 (2015).

[9] S. Guerber, C. Alonso-Ramos, D. Benedikovic, E. DuránValdeiglesias, X. Le Roux, N. Vulliet, E. Cassan, D. MarrisMorini, C. Baudot, F. Boeuf, and L. Vivien, IEEE Photon. Technol. Lett. 30, 1679 (2018).

[10] C.-C. Huang, Sci. Rep. 8, 17338 (2018).

[11] L. Xu, Y. Wang, E. El-Fiky, D. Mao, A. Kumar, Z. Xing, M. G. Saber, M. Jacques, and D. V. Plant, J. Lightw. Technol. 37, 1231 (2019).

[12] B. Shen, P. Wang, R. Polson, and R. Menon, Nat. Photon. 9, 378 (2015).

[13] H. Xu, D. Dai, and Y. Shi, Laser Photon. Rev. 13, 1800349 (2019).

[14] S. Longhi, Laser Photon. Rev. 3, 243 (2009).

[15] N. V. Vitanov, A. A. Rangelov, B. W. Shore, and K. Bergmann, Rev. Mod. Phys. 89, 015006 (2017).

[16] E. Paspalakis, Opt. Commun. 258, 30 (2006).

[17] S. Longhi, G. Della Valle, M. Ornigotti, and P. Laporta, Phys. Rev. B 76, 201101(R) (2007).

[18] F. Dreisow, M. Ornigotti, A. Szameit, M. Heinrich, R. Keil, S. Nolte, A. Tünnermann, and S. Longhi, Appl. Phys. Lett. 95, 261102 (2009).

[19] R. Menchon-Enrich, A. Llobera, J. Vila-Planas, V. J. Cadarso, J. Mompart, and V. Ahufinger, Light Sci. Appl. 2, e90 (2013).

[20] C. Ciret, V. Coda, A. A. Rangelov, D. N. Neshev, and G. Montemezzani, Phys. Rev. A 87, 013806 (2013).

[21] H. Oukraou, L. Vittadello, V. Coda, C. Ciret, M. Alonzo, A. A. Rangelov, N. V. Vitanov, and G. Montemezzani, Phys. Rev. A 95, 023811 (2017).

[22] X. Xiong, C.-L. Zou, X.-F. Ren, and G.-C. Guo, Opt. Express 21, 17097 (2013).

[23] P. Aashna and K. Thyagarajan, J. Opt. 19, 065805 (2017).

[24] Y.-P. Liao and R.-C. Lu, IEEE J. Sel. Top. Quantum Electron. 6, 88 (2000).

[25] A. Yariv, IEEE J. Quantum Electron. 9, 919 (1973).

[26] K. Okamoto, in Fundamentals of Optical Waveguides, 2nd ed., edited by K. Okamoto (Academic, Burlington, 2006), pp. 329-397.

[27] N. V. Vitanov, M. Fleischhauer, B. W. Shore, and K. Bergmann, Adv. At. Mol. Phys. 46, 55 (2001).

[28] A. Yariv, Optical Electronics in Modern Communications (Oxford University Press, Oxford, 1997).

[29] M. Lawrence, Rep. Prog. Phys. 56, 363 (1993).
[30] M. Bazzan and C. Sada, Appl. Phys. Rev. 2, 040603 (2015).

[31] R. V. Schmidt and I. P. Kaminow, Appl. Phys. Lett. 25, 458 (1974).

[32] J. L. Jackel, C. E. Rice, and J. J. Veselka, Appl. Phys. Lett. 41, 607 (1982).

[33] M. De Micheli, J. Botineau, P. Sibillot, D. B. Ostrowski, and M. Papuchon, Opt. Commun. 42, 101 (1982).

[34] M. De Micheli, J. Botineau, S. Neveu, P. Sibillot, D. B. Ostrowski, and M. Papuchon, Opt. Lett. 8, 114 (1983).

[35] D. Zang and C. Tsai, Appl. Phys. Lett. 46, 703 (1985).

[36] V. P. Hinkov and E. Ise, J. Lightw. Technol. 4, 444 (1986).

[37] A. L. Dawar, S. M. Al-Shukri, R. M. De La Rue, A. C. G. Nutt, and G. Stewart, Opt. Commun. 61, 100 (1987).

[38] H. Ma, A. K.-Y. Jen, and L. R. Dalton, Adv. Mater. 14, 1339 (2002).

[39] M. C. Oh, M. H. Lee, and H. J. Lee, IEEE Photon. Technol. Lett. 11, 1144 (1999).

[40] J. W. Kim, S. H. Park, W. S. Chu, and M. C. Oh, Opt. Express 20, 12443 (2012).

[41] M. K. Szczurowski, T. Martynkien, G. Statkiewicz-Barabach, W. Urbanczyk, L. Khan, and D. J. Webb, Opt. Lett. 35, 2013 (2010).

[42] Y. Q. Shi, W. H. Steier, L. P. Yu, M. Chen, and L. R. Dalton, Appl. Phys. Lett. 59, 2935 (1991).

[43] Ph. Dittrich, G. Montemezzani, P. Bernasconi, and P. Günter, Opt. Lett. 24, 1508 (1999).

[44] M. Gorram, V. Coda, P. Thévenin, and G. Montemezzani, Appl. Phys. B 95, 565 (2009).

[45] V. Coda, C. Ciret, T. Mengis, M. Gorram, G. Montemezzani, M. Chauvet, and F. Devaux, Opt. Commun. 355, 382 (2015).

[46] M. Klotz, H. X. Meng, G. J. Salamo, M. Segev, and S. R. Montgomery, Opt. Lett. 24, 77 (1999).

[47] R. S. Cudney, J. Fousek, M. Zgonik, P. Günter, M. H. Garrett, and D. Rytz, Appl. Phys. Lett. 63, 3399 (1993).

[48] M. Jazbinšek and M. Zgonik, Appl. Phys. B 74, 407 (2002).

[49] M. Zgonik, R. Schlesser, I. Biaggio, E. Voit, J. Tscherry, and P. Günter, J. Appl. Phys. 74, 1287 (1993).

[50] M. Zgonik, P. Bernasconi, M. Duelli, R. Schlesser, P. Günter, M. H. Garrett, D. Rytz, Y. Zhu, and X. Wu, Phys. Rev. B 50, 5941 (1994).

[51] D. C. Zografopoulos, R. Asquini, E. E. Kriezis, A. d'Alessandro, and R. Beccherelli, Lab Chip 12, 3598 (2012).

[52] J. Beeckman, R. James, F. A. Fernandez, W. De Cort, P. J. M. Vanbrabant, and K. Neyts, J. Lightw. Technol. 27, 3812 (2009).

[53] W. De Cort, J. Beeckman, T. Claes, K. Neyts, and R. Baets, Opt. Lett. 36, 3876 (2011). 